\title{
Arbeitszeitpolitische Kontroversen im Spiegel der Arbeitszeitwünsche
}

\author{
Wie soll die zukünftige Arbeitszeit aussehen? Die vielfältigen Antworten auf diese Frage \\ gehen weit auseinander. Übereinstimmung besteht lediglich in der Einschätzung, dass die \\ gegenwärtigen Arbeitszeitstrukturen für die Beschäftigten suboptimal sind. Die Arbeits- \\ zeitpolitik steht an einem Scheideweg. Um die Richtungsfindung zu erleichtern, zeigt der \\ Beitrag auf, welche Arbeitszeiten die Beschäftigten favorisieren - immer unter der Vor- \\ aussetzung entsprechend angepasster Einkommen. Eine Minderheit, vor allem teilzeitbe- \\ schäftigte Frauen, befürwortet längere, die Mehrheit, vor allem vollzeitbeschäftigte Män- \\ ner und Frauen, wünscht kürzere Arbeitszeiten. ${ }^{\bullet}$
}

ELKE HOLST, HARTMUT SEIFERT

\section{Problemstellung}

In der Arbeitszeitdebatte zeichnet sich eine neue Kontroverse ab. Es geht um die Frage, ob zukünftig wieder länger oder kürzer gearbeitet werden soll. In der ersten Alternative sieht die Bundesagentur für Arbeit (BA) (2011) einen Weg, das in den nächsten Jahren aus demografischen Gründen knapper werdende Arbeitskräfteangebot und speziell den drohenden Fachkräftemangel zu lindern. Diesem Vorschlag stehen sehr unterschiedlich begründete Überlegungen und Konzepte gegenüber, die eher auf kürzere Arbeitszeiten hinauslaufen. Sie verfolgen familienund gleichstellungspolitische Ziele, sollen alternsgerechtes Arbeiten fördern, die Chancen auf Work-Life-Balance verbessern oder vermehrte Zeitautonomie ermöglichen. Ungeachtet der spezifischen Zielsetzungen und ihrer Begründungszusammenhänge ist diesen Ansätzen gemeinsam, dass sie neue Arbeitszeitarrangements anstreben, die den Beschäftigten mehr Spielraum einräumen, ihre privaten Zeitanforderungen mit den betrieblichen auszubalancieren, und Optionen bieten, die Dauer der Arbeitszeit entsprechend den sich ändernden Präferenzen variieren zu können.

Vor diesem Hintergrund steht Arbeitszeitpolitik vor der grundsätzlichen Entscheidung, zukünftig entweder den Weg zu kürzeren Arbeitszeiten in Verbindung mit vermehrten Optionen für individuelle Arbeitszeitgestaltung fortzusetzen oder zu längeren Arbeitszeiten zurückzukehren. Will man einzelne zeitpolitische Ziele und Konzepte nicht a priori ausschließen, dann ist es für die Entscheidungsfindung hilfreich, folgenden Fragen nachzugehen: Erstens ist zu klären, ob die vorgeschlagenen Ausweitungen der Arbeitszeiten tatsächlich die erhofften beschäftigungspolitischen Effekte erwarten lassen und als zielführend anzusehen sind. Zweitens stellt sich die Frage, inwieweit generell verlängerte Arbeitszeiten den Zeitpräferenzen der Beschäftigten entsprechen. Andernfalls wären Wege aufzuzeigen, wie sich verlängerte Arbeitszeiten, sollen sie nicht kollektiv verordnet werden, beispielsweise durch Anreize erreichen ließen.

Im Mittelpunkt des nachfolgenden Beitrages stehen die Zeitwünsche der Beschäftigten (Abschnitt 3). Sie liefern Orientierungspunkte für zeitpolitische Konzepte. Empirisch wird untersucht, wie die gewünschten zu den tatsächlich geleisteten sowie zu den vereinbarten Arbeitszeiten stehen und wie sich diese Zeitgrößen im Zeitablauf verändert haben. Außerdem wird gezeigt, mit welchen Veränderungen des Beschäftigungspotenzials zu rechnen ist, wenn die Beschäftigten ihre Zeitpräferenzen realisieren

Wir danken Lea Kröger, studentische Hilfskraft am Deutschen Institut für Wirtschaftsforschung (DIW) Berlin, für ihre Unterstützung bei der Aufbereitung der Daten. 
könnten und auch würden (4). Bevor diesen Fragen nachgegangen wird, soll kurz die aktuelle Arbeitszeitdebatte rekapituliert werden (2). In diesem Zusammenhang werden die von verlängerten Arbeitszeiten erhofften Effekte auf das Arbeitsangebot hinterfragt. Ein knappes Fazit (5) rundet den Beitrag ab.

Der analytische Blick richtet sich allein auf die Dauer der Arbeitszeit; die beiden anderen Dimensionen, Lage und Verteilung der Arbeitszeit, die als wichtige Einflussfaktoren für die präferierte Arbeitszeitdauer anzusehen sind, bleiben in der nachfolgenden Analyse ausgeklammert.

\section{Arbeitszeitpolitische Diskussionen}

\subsection{Der Stellenwert verkürzter und flexibler Arbeitszeiten}

In den letzten Jahren war die Arbeitszeit zwar stets Gegenstand gesellschafts-, sozial- und arbeitspolitischer Debatten. Auf die Arbeitszeitentwicklung haben sie aber nur geringen Einfluss ausgeübt. Die Diskussionen wurden in aller Regel aus jeweils isolierter Zielperspektive geführt, ohne dass die konzeptionellen Stränge zusammengebracht, in ein stimmiges Konzept integriert, Prioritäten gesetzt und in entsprechenden Maßnahmen umgesetzt wurden. Im Mittelpunkt der zeitpolitischen Debatten standen die in der bisherigen Realisierung als unzureichend angesehenen gesellschafts- und sozialpolitischen Großprojekte der besseren Vereinbarkeit von Beruf und Familie, der Geschlechtergerechtigkeit, des alternsgerechten Arbeitens und der Sicherung der Arbeitsfähigkeit/Gesundheit, der vermehrten Weiterbildungszeit und des Wunsches nach größerer Zeitsouveränität. Auch ging es um die Rolle, die variable Arbeitszeiten zur Sicherung der Beschäftigung im Konjunkturzyklus spielen können. ${ }^{2}$ Über diese Ziele bestand und besteht im Grundsatz ein breiter gesellschaftlicher Konsens. Gleichwohl hat es in den letzten Jahren nur wenige Vorstöße gegeben, die Arbeitszeit solchen Zielsetzungen entsprechend neu auszurichten. $\mathrm{Zu}$ den Ausnahmen zählen, sieht man einmal von zahlreichen betrieblichen Vereinbarungen ab, die Demografie-Tarifverträge in der Stahl- und in der Chemischen Industrie, das 2008 in Kraft getretene Pflegezeitgesetz sowie das ab 2009 geltende „Gesetz zur Verbesserung der Rahmenbedingungen für die Absicherung flexibler Arbeitszeitregelungen“ (Flexi-II-Gesetz). Diese Vereinbarungen berühren primär die Verteilung der jeweils kollektiv oder individuell vereinbarten Arbeitszeitdauer und nur indirekt in einer langfristigen Perspektive auch Veränderungen der jeweiligen Dauer der Regelarbeitszeit, die sich über die gesamte Erwerbsbiografie erstrecken kann.

Häufig bleiben die aus den genannten Zielen abgeleiteten arbeitszeitpolitischen Vorschläge vage, formulieren keine quantifizierten Zielgrößen für Änderungen in der Ar- beitszeitdauer, sondern beschränken sich auf nicht weiter konkretisierte Grundkonzepte, wie z. B. Wahlarbeitszeiten, familienfreundliche "Arbeitszeitoptionsmodelle“ oder mehr Zeitsouveränität im Alltag sowie entlang des Lebenslaufs (Sachverständigenkommission 2011). Ähnlich allgemein lauten die Vorschläge im Fünften Altenbericht, die für eine flexible Arbeitszeitgestaltung plädieren, die von bezahltem Erziehungsurlaub bis hin zu Ansprüchen auf unbezahlte Verringerung der Arbeitszeit für beide Geschlechter reicht (BMFSFJ 2005). Kaum konkreter fallen die Vorschläge im Achten Familienbericht aus. Sie sprechen sich für eine Umverteilung der Zeitressourcen zwischen den Geschlechtern und im Lebenslauf im Sinne der Präferenzen aller Beteiligten aus (BMFSFJ 2011).

In keinem dieser Konzepte ist die Rede von längeren Arbeitszeiten. Offenbar gelten längere Arbeitszeiten, wenngleich nicht explizit thematisiert, als nicht zielführend. Die vorgeschlagenen Ansätze weisen entweder in Richtung auf kürzere Arbeitszeiten, zumindest für Vollzeitbeschäftigte und vor allem für diejenigen mit sehr langen Wochenarbeitszeiten, oder aber sie plädieren für präferenzgerechte Wahlarbeitszeiten.

\subsection{Effekte verlängerter Arbeitszeiten}

Konkretere Aussagen leiten sich aus verschiedenen Modellrechnungen zur Abschätzung der Potenzialeffekte von veränderten Arbeitszeitdauern ab. ${ }^{\mathbf{0}}$ Hierzu gehören die Schätzungen von Eichhorst et al. (2011) zu Wunscharbeitszeiten. Sie bilden den Ausgangspunkt für ein Konzept der familienfreundlichen Arbeitszeiten. Für die Gruppe der erwerbstätigen Mütter mit Kindern bis zu 16 Jahren schätzen die Autoren, mit welchen zusätzlichen Beschäftigungspotenzialen bei einer präferenzgerechten Arbeitszeitdauer zu rechnen ist. Die Wünsche nach längeren Arbeitszeiten, gemessen in Stundenvolumina, überwiegen die nach kürzeren. Bilanzierend ergibt sich ein Arbeitsangebotseffekt von zusätzlich 75.000 vollzeitäquivalenten Stellen. Diese Modellrechnungen beziehen sich allerdings nicht auf sämtliche Beschäftigte, sondern nur auf die genannte Teilgruppe, die besonderen familialen Zeitanforderungen ausgesetzt ist und deshalb entsprechende Zeitverwendungsmöglichkeiten reklamiert.

Ebenfalls nur für eine Teilgruppe der Beschäftigten berechnet Wanger (2011) das zusätzliche Arbeitspotenzial, das sich ergeben könnte, wenn teilzeitbeschäftigte Frauen ihre Wünsche nach verlängerten Arbeitszeiten realisieren könnten. Dadurch ließe sich das Arbeitsvolumen rechnerisch um etwa eine Million Vollzeitäquivalente steigern (Wanger

2 Vgl. hierzu vor allem die Beiträge in Seifert 2005; Groß/ Seifert 2010

(3) Derartige mechanische Modellrechnungen standen lange Zeit in der Kritik, als sie dazu dienten, die Beschäftigungseffekte von tariflichen Arbeitszeitverkürzungen abzuschätzen. 
2011, S. 6). Diese Modellrechnung ist allerdings selektiv. Ausgeblendet bleiben vor allem die Verkürzungswünsche der Vollzeitbeschäftigten, sowohl der Frauen als auch der Männer. Gerade aber für diese zahlenmäßig größte Gruppe der Beschäftigten lässt sich, wie noch zu zeigen ist, ein beachtliches Verkürzungspotenzial errechnen. Außerdem gehen die Berechnungen von der Differenz zwischen vereinbarten und gewünschten Arbeitszeiten aus. Realistischer für derartige Potenzialschätzungen ist aber, die Differenz zwischen gewünschten und tatsächlichen Arbeitszeiten zum Ausgangspunkt zu nehmen. Da Letztere durchschnittlich deutlich über den vereinbarten Größen liegen, fällt die Zeitdifferenz, die als Berechnungsgrundlage für die Potenzialschätzung dient, kleiner aus. Der rechnerische Potenzialeffekt schrumpft.

Aus einer völlig anderen Perspektive schätzen Modellrechnungen der BA (2011) die Potenzialeffekte von Arbeitszeitänderungen. Ausgangspunkt bilden nicht die Wünsche der Beschäftigten, sondern allein Überlegungen, wie sich das Arbeitspotenzial steigern ließe. Angenommen wird eine generelle Ausweitung der Wochenarbeitszeit um $5 \%$ oder gut zwei auf 44 Stunden (Std.) für Vollzeitbeschäftigte. Dieser Schritt soll das Arbeitsvolumen um bis zu gut einer Million Vollzeitäquivalente steigern. Vorgeschlagen ist ferner, Anreize zu geben, um den tariflichen Jahresurlaub zu verkürzen. Zusätzliches Potenzial wird außerdem in der Teilzeitarbeit von Frauen gesehen. Denn in Deutschland leistet ein nicht nur im Vergleich zu den übrigen europäischen Ländern relativ hoher Anteil der Frauen Teilzeitarbeit. Deren durchschnittliche Arbeitszeit ist außerdem mit nur 18,5 Wochenstunden die kürzeste in Europa (BA 2011). Arbeitszeitverlängerungen, entweder durch eine Aufstockung der Teilzeitstunden oder durch eine partielle Umwandlung von Teilzeit- in Vollzeitstellen könnten, so die Schätzungen der BA, das Arbeitspotenzial um bis zu einer weiteren Million Vollzeitäquivalente steigern. In der Summe lassen die verschiedenen Möglichkeiten, die Arbeitszeit zu verlängern, ein beachtliches Potenzial an zusätzlichem Arbeitsangebot erwarten und werden aus Sicht der BA somit zu einer wichtigen Stellgröße, um drohende Engpasssituationen am Arbeitsmarkt zu vermeiden.

Diese Modellrechnungen der BA haben allerdings mechanischen Charakter, blenden Rückkoppelungseffekte ebenso völlig aus wie notwendige infrastrukturelle Rahmenbedingungen, wie beispielsweise die Versorgung mit Kinderbetreuungseinrichtungen. Sie lassen ferner die Arbeitszeitwünsche der Beschäftigten außer Acht, ganz abgesehen von den Arbeitszeitprofilen, die die Nachfrageseite anbietet. Deshalb zeichnen sie ein eher unrealistisches Bild und überschätzen die zusätzlich mobilisierbaren Beschäftigungspotenziale. An Gewicht gewinnt dieser Einwand, da die Modellrechnungen nicht-intendierte Effekte unberücksichtigt lassen. So dürften verlängerte Wochenarbeitszeiten unter ansonsten unveränderten Arbeitsbedingungen, vor allem bei gegebener Arbeitsintensität, das Risiko erhöhen, dass noch mehr Beschäftigte aus gesundheitlichen Gründen ihre
Erwerbstätigkeit vorzeitig aufgeben müssen. So erfolgten 2010 gut $21 \%$ aller Rentenzugänge wegen verminderter Erwerbsfähigkeit (DRV 2011). Das durchschnittliche Rentenzugangsalter lag bei gut 50 Jahren. Diese Werte dürften sich verschlechtern, da zu der verlängerten Arbeitszeit weitere Belastungen durch den ungebrochenen Trend zu vermehrter Schicht- und Nachtarbeit ${ }^{\boldsymbol{\Phi}}$ hinzukämen. Außerdem beeinflussen Diskrepanzen zwischen tatsächlichen und gewünschten Arbeitszeiten die Gesundheit negativ, wenn die erste Größe dauerhaft die zweite übertrifft (Bell et al. 2011). Nicht-präferenzgerechte Arbeitszeitverlängerungen würden diesen Effekt verstärken. Kumulativ erhöhte Gesamtbelastungen würden sich entsprechend negativ auf das Erwerbspersonenpotenzial auswirken. Die Zahl der Erwerbsminderungsrenten dürfte zunehmen und das durchschnittliche Zugangsalter weiter sinken. Das Arbeitskräftepotenzial würde schrumpfen und nicht steigen. Diesen Effekt könnten vermehrte Krankentage infolge dauerhaft erhöhter Arbeitsbelastungen noch verstärken.

Längere Arbeitszeiten erschweren außerdem die Vereinbarkeit von Beruf und Familie. Bei gegebener geschlechtsspezifischer Aufgabenteilung im Haushalt und den begrenzten Betreuungsmöglichkeiten könnten sich vor allem Mütter zunehmend gezwungen sehen, von (der dann erhöhten) Vollzeit auf Teilzeit umzusteigen - insbesondere, wenn männliche Partner angesichts verlängerter Arbeitszeiten noch weniger Zeit für familiäre Aufgaben aufbringen (könnten). Zudem wird eine Aufstockung der Stunden der oft teilzeitbeschäftigten Partnerinnen damit erschwert.

Diesen möglichen Reaktionen steht entgegen, dass sich die Opportunitätskosten einer Nichterwerbstätigkeit durch das gestiegene Humankapital von Frauen erhöht haben. Abzuwarten bleibt, wie die Konflikte um die Vereinbarkeit von Beruf und Familie bei verlängerten Arbeitszeiten gelöst werden. Wenig wahrscheinlich ist, dass eine weitere Ausdehnung der Arbeitszeit die Zeitkultur in den Unternehmen durch flexiblere Arbeitszeiten für Frauen und Männer verbessert.

Verlängerte Arbeitszeiten erlauben außerdem weniger Zeit für berufliche Weiterbildung in Eigenregie. Beruflicher Aufstieg und Wechsel in chancenreichere und besser entlohnte Tätigkeiten gelingen weniger reibungslos oder unterbleiben. Das erschwert den notwendigen Strukturwandel. Schließlich schwindet mit verlängerten Regelarbeitszeiten der Spielraum, mit Hilfe von Zeitkonten die Arbeitszeit mit zyklischen Nachfrageschwankungen zu synchronisieren, wie dies in der Krise 2008/09 der Fall war. Wenn im konjunkturellen Abschwung die Arbeitszeit zur Sicherung der Beschäftigung zurückgenommen wird, muss sie im nachfolgenden Aufschwung im gleichen Umfang über den vereinbarten Durchschnittswert verlängert werden, was bei langen Regelarbeitszeiten eine überlange Arbeitswoche mit entspre-

42010 leisteten 17,2 \% der Beschäftigten Schichtarbeit, während es 1991 12,7 \% waren (Statistisches Bundesamt 2011). 
chenden Folgen für Arbeitsproduktivität, Regenerationsfähigkeit usw. zur Folge hätte. Insgesamt sind unter diesen Vorzeichen die Schätzwerte der BA nach unten zu korrigieren.

\section{Arbeitszeiten zwischen Wunsch und Wirklichkeit}

\subsection{Daten}

Die nachfolgenden empirischen Befunde basieren auf dem Sozio-oekonomischen Panel (SOEP). Das SOEP ist eine repräsentative Wiederholungsbefragung, die bereits seit 1984 läuft. Im Auftrag des Deutschen Instituts für Wirtschaftsforschung (DIW) Berlin werden derzeit über 20.000 Personen aus rund 11.000 Haushalten von TNS Infratest Sozialforschung befragt (Wagner et al. 2007). Aufgrund von Ausfällen von Personen („Panelmortalität“) und Aufstockungen der Stichprobe sind die Fallzahlen im Zeitverlauf nicht konstant. Im Anfangsjahr des hier relevanten Untersuchungszeitraums von 1993 bis 2009 lagen Informationen zu insgesamt mehr als 13.000 Personen vor, im Endjahr zu mehr als 21.000 Personen. Die Veränderungen in der Stichprobengröße werden bei Gewichtung und Hochrechnung des SOEP berücksichtigt (Kroh 2010). Die Daten geben Auskunft zu zahlreichen arbeitsmarktrelevanten Größen, so auch über die tatsächliche, gewünschte und vereinbarte Arbeitszeit.

\subsection{Zeitgrößen}

Bei der Dauer der Arbeitszeit ist zwischen drei Größen zu unterscheiden: der vereinbarten, der tatsächlichen und der gewünschten ${ }^{\ominus}$ Arbeitszeit. Im arbeitszeitpolitischen Idealfall stimmen die Werte der drei Größen überein, aber auch das Gegenteil ist denkbar. Je nachdem, in welchem Maße diese Größen voneinander abweichen, unterscheiden sich die Ausgangsbedingungen und Anforderungen für arbeitszeitpolitisches Handeln. Weisen die Arbeitszeitpräferenzen in Richtung kürzerer Arbeitszeiten, ist eine auf Verlängerung abzielende Zeitpolitik mit anderen Herausforderungen konfrontiert als bei Kongruenz beider Arbeitszeitgrößen. Das gilt umgekehrt natürlich auch für den Fall, dass längere Arbeitszeiten gewünscht werden, politische Konzepte aber in die Gegenrichtung zielen. Wie nun stehen die drei Größen der Arbeitszeitdauer zueinander und wie haben sie sich seit 1993 verändert?

\subsection{Beschäftigte insgesamt}

Vereinbarte und tatsächliche Arbeitszeiten von allen Beschäftigten, Vollzeit- und Teilzeit eingeschlossen, klaffen auseinander (Tabelle 1). Männer und Frauen arbeiten durchschnittlich länger, als es ihre kollektiven oder individuellen Vereinbarungen vorsehen. Besonders groß mit durchschnittlich 4,5 Std. - auf den gesamten Beobachtungszeitraum bezogen - fallen die Differenzen bei Männern aus, bei Frauen sind es 1,8 Std., 2009 waren es 4,3 bzw. 2,1 Std. Sowohl bei den Frauen als auch bei den Männern sind die Abstände zwischen beiden Größen gegenüber 1993 leicht gewachsen. Dabei ist in Rechnung zu stellen, dass aufgrund des scharfen Konjunktureinbruchs 2009 die durchschnittliche Arbeitszeit erheblich abgesenkt wurde, um bedrohte Arbeitsverhältnisse zu sichern (Herzog-Stein/Seifert 2010). ${ }^{\bullet}$ Die Arbeitszeit von vollzeitbeschäftigten Männern dürfte hierdurch stärker gesunken sein als die von Frauen, weil Erstere überproportional in den besonders von der Wirtschaftskrise betroffenen Bereichen des Produzierenden Gewerbes beschäftigt sind.

Ähnliche Zeitdiskrepanzen zeigen sich auch zwischen der tatsächlichen und der gewünschten Dauer der Arbeitszeit (Tabelle 1). Die Arbeitszeitwirklichkeit ist teilweise weit von den gewünschten Größen der Beschäftigten entfernt. Beschäftigte mit einer nicht wunschgerechten Arbeitszeit sind offensichtlich gehindert, die für sie optimale Kombination von Zeit- und Geldnutzen zu realisieren und damit den Gesamtnutzen ihrer Arbeit zu steigern. Um sich dem nutzenmaximalen Verhältnis von Einkommen und nichterwerbsgebundener Zeit ${ }^{\boldsymbol{0}}$ anzunähern, sind die Unterschiede zwischen tatsächlichen und gewünschten Arbeitszeiten zu verkleinern. Vorausgesetzt ist dabei, dass angestrebte Veränderungen in der Dauer der Arbeitszeit proportional auch die Einkommenshöhe verändern.

Die Diskrepanz zwischen tatsächlicher und gewünschter Arbeitszeit ist seit 1993 zwar kleiner geworden, gleichwohl hat sich die Schere weder bei Frauen und noch weniger bei Männern geschlossen. Frauen favorisieren - auf den gesamten Beobachtungszeitraum bezogen und im Saldo aller Zeitwünsche - eine um durchschnittlich 2,6 Std. pro Woche kürzere Arbeitszeit, und zwar selbst dann, wenn sich auch ihr Einkommen entsprechend verringern würde. 1993 lag die Differenz bei 4,1 Std., 2009 waren es nur noch 1,6 Std. Bei Männern klaffen Wunsch und Wirklichkeit wesentlich

(5) Zeitwünsche werden in aller Regel von Handlungsrestriktionen wie geschlechtsspezifische Arbeitsteilungen, Angebote an öffentlicher Kinderbetreuung, Einkommen usw. beeinflusst. Diese Aspekte müssen hier ausgeblendet bleiben.

(6 Die beiden betrachteten Jahre 1993 und 2009 sind unter konjunkturellen Aspekten in etwa vergleichbar, da beide von Rezessionen mit durchschnittlich kürzeren Arbeitszeiten als in Boomjahren geprägt sind. Der Abbau von Zeitguthaben und die Bildung von Zeitschulden, die Reduzierung der Wochenarbeitszeit im Rahmen von tariflichen Korridormodellen sowie Kurzarbeit hatten zu einem starken Rückgang der geleisteten Arbeitszeit geführt (Herzog-Stein/ Seifert 2010).

(7) Gemeint ist damit die gesamte nicht-erwerbsgebundene Zeit, die für Reproduktions-, Haushalts-, Familien- und Betreuungs- sowie soziale Aktivitäten einschließlich Ehrenamt verwendet wird. 
weiter auseinander, die Differenz lag 2009 bei 3,6 Std. pro Woche, während es 1993 sogar 4,2 Std. waren. Sowohl bei Frauen als auch Männern weisen die durchschnittlichen Zeitwünsche in dem hier betrachteten Zeitraum leicht nach oben. Für die Robustheit der Daten spricht, dass die Jahreswerte nur geringe Schwankungen aufweisen.

Die deutliche Annäherung der gewünschten an die tatsächlichen Arbeitszeiten der Frauen seit 1993 erfolgte von beiden Seiten: Die präferierten Wochenarbeitszeiten stiegen um durchschnittlich 0,8 Std., gleichzeitig sanken die Werte der tatsächlichen um 1,8 Std. Für die Vermutung, dass der Rückgang der tatsächlichen Arbeitszeit auf das 2001 in Kraft getretene Teilzeit- und Befristungsgesetz (TzBfG) zurückgehen könnte, liefern die Arbeitszeitdaten keine Anhaltspunkte. Erstaunlich ist vielmehr, dass die durchschnittliche tatsächliche Arbeitszeit seit 2001 unverändert geblieben ist, wenn man von kleineren zwischenzeitlichen Variationen absieht. Bei der Annäherung von gewünschten und tatsächlichen Arbeitszeiten sieht es vielmehr so aus, als ob es sich um eine von den rechtlichen Ansprüchen auf individuelle Arbeitszeitverkürzung weitgehend unabhängige Entwicklung handelt, denn bereits bis 2000, also kurz vor Inkrafttreten des rechtlichen Anspruchs auf Verkürzung der Arbeitszeit, war die Differenz zwischen tatsächlichen und gewünschten Arbeitszeiten bei Frauen deutlich von 4,1 (1993) auf 2,8 Std. geschrumpft. Diese Veränderung geht vor allem auf die im Schnitt geringeren tatsächlichen Arbeitszeiten der immer mehr in Teilzeit tätigen Frauen im Osten und den allgemein bei den Frauen gestiegenen Arbeitszeitwünschen im Westen zurück (siehe auch Abschnitte 3.5 und 4 ).

Um den arbeitszeitpolitischen Handlungsbedarf identifizieren zu können, ist es notwendig, neben den tatsächlichen und den gewünschten auch noch die vereinbarten Arbeitszeiten in die Betrachtung einzubeziehen (Tabelle 1). Dabei zeigt sich, dass die gewünschten Arbeitszeiten wesentlich näher an den vereinbarten als an den tatsächlichen Größen liegen. Insofern treffen die kollektiv und individuell vereinbarten Arbeitszeiten die Wünsche der Beschäftigten wesentlich genauer als die betrieblichen Wirklichkeiten. Männer favorisierten 2009 eine Arbeitszeit, die um durchschnittlich 0,7 Std. und bei Frauen um 0,5 Std. über den vereinbarten Größen lag. Gegenüber 1993 hat sich das Bild beträchtlich geändert. Damals lag die von Frauen gewünschte um durchschnittlich 2,5 Std. unter der vereinbarten Arbeitszeit; bei Männern waren beide Größen in etwa deckungsgleich. Seitdem ist die durchschnittlich vereinbarte Arbeitszeit von Frauen um über 2 Std. gesunken, während gleichzeitig die Wünsche etwas längere Arbeitszeiten nahelegen. Ähnlich, wenngleich auf höherem Niveau, sehen die Änderungen bei den Zeitwünschen der Männer aus, die vereinbarte Arbeitszeit blieb jedoch weitgehend konstant. Geht man von den durchschnittlichen Zeitwünschen der Beschäftigten aus, dann würde sich deren Zeitnutzen beträchtlich steigern lassen, wenn es gelänge, die tatsächlichen mit den tarif- oder individualvertraglich vereinbarten Ar-
TABELLE 1

\section{Vereinbarte, tatsächliche und gewünschte Arbeitszeit von abhängig beschäftigten* Männern und Frauen in Deutschland 1993-2009}

in Wochenstunden

\begin{tabular}{|c|c|c|c|c|c|c|}
\hline & \multicolumn{3}{|c|}{ Frauen } & \multicolumn{3}{|c|}{ Männer } \\
\hline & $\begin{array}{l}\text { Vereinbarte } \\
\text { Arbeitszeit }\end{array}$ & $\begin{array}{l}\text { Tatsächliche } \\
\text { Arbeitszeit }\end{array}$ & $\begin{array}{l}\text { Gewünschte } \\
\text { Arbeitszeit }\end{array}$ & $\begin{array}{l}\text { Vereinbarte } \\
\text { Arbeitszeit }\end{array}$ & $\begin{array}{l}\text { Tatsächliche } \\
\text { Arbeitszeit }\end{array}$ & $\begin{array}{l}\text { Gewünschte } \\
\text { Arbeitszeit }\end{array}$ \\
\hline 1993 & 32,4 & 34,0 & 29,9 & 38,4 & 42,5 & 38,3 \\
\hline 1994 & 32,0 & 33,7 & 30,2 & 38,4 & 42,5 & 38,4 \\
\hline 1995 & 31,6 & 33,5 & 28,8 & 38,0 & 42,7 & 37,7 \\
\hline $1996^{* *}$ & 31,5 & 33,0 & 29,5 & 38,0 & 42,7 & 38,2 \\
\hline 1997 & 31,5 & 33,4 & 30,2 & 38,1 & 42,9 & 38,7 \\
\hline 1998 & 31,3 & 33,5 & 30,2 & 37,9 & 42,8 & 37,6 \\
\hline 1999 & 30,9 & 32,6 & 29,8 & 38,1 & 42,6 & 38,2 \\
\hline 2000 & 30,8 & 32,3 & 29,5 & 38,1 & 42,7 & 38,4 \\
\hline 2001 & 30,2 & 32,2 & 29,9 & 38,0 & 42,7 & 38,4 \\
\hline 2002 & 29,9 & 31,3 & 29,1 & 38,1 & 42,7 & 38,4 \\
\hline 2003 & 29,6 & 31,4 & 29,5 & 37,8 & 42,2 & 38,3 \\
\hline 2004 & 30,1 & 31,5 & 30,0 & 38,0 & 42,3 & 38,7 \\
\hline 2005 & 30,3 & 31,7 & 30,3 & 38,3 & 42,6 & 39,0 \\
\hline 2006 & 29,9 & 31,7 & 30,3 & 38,3 & 42,7 & 38,8 \\
\hline 2007 & 29,9 & 31,8 & 30,5 & 38,5 & 43,2 & 39,2 \\
\hline 2008 & 30,1 & 32,1 & 30,1 & 38,5 & 42,7 & 38,7 \\
\hline 2009 & 30,1 & 32,2 & 30,6 & 38,5 & 42,8 & 39,2 \\
\hline
\end{tabular}

* Abhängig Beschäftigte = Angestellte, Arbeiter, Beamte (ohne Auszubildende), gewichtete Mittelwerte. ** Für 1996 liegen keine Angaben zu den gewünschten Arbeitszeiten vor, daher geschätzt (Mittelwert Differenz zwischen 1995 und 1997).

Quelle: SOEP 2009; Berechnungen der Autoren.

beitszeiten in Einklang zu bringen. Ein wesentlicher Schritt wäre getan, wenn Überstunden und Mehrarbeit vermieden und die vereinbarten Arbeitszeiten eingehalten würden.

\subsection{Vollzeitbeschäftigte}

Ein anderes Bild zeigt sich, wenn man die Beschäftigten grob nach den Kategorien Vollzeit- und Teilzeit differenziert. Die vereinbarte Arbeitszeit von Vollzeitbeschäftigten hat sich zwischen 1993 und 2009 in beiden Teilen Deutschlands leicht gegenläufig entwickelt. Die Annäherung geht von beiden Seiten aus. In Westdeutschland ist die vereinbarte Arbeitszeit bei Männern um durchschnittlich 0,9 und bei Frauen um 0,3 Std. gestiegen, in Ostdeutschland dagegen bei den Frauen um 0,7 und bei den Männern um 0,2 Std. gesunken (Abbildung 1). Die Männer in den alten Bundesländern arbeiten 2009 durchschnittlich etwa 5 Std. länger, als es ihre Vereinbarungen vorsehen. 1993 war die Differenz mit 4 Std. etwas kleiner, in Ostdeutschland mit 5,5 Std. dagegen etwas größer.

Die langen Arbeitszeiten von vollzeitbeschäftigten Männern liegen deutlich, zumindest wenn man Durchschnittswerte zugrunde legt, über deren Zeitpräferenzen. Diese Beschäftigtengruppe wünscht, durchschnittlich 
um 5,2 Std. in Ost- und 4,7 Std. in Westdeutschland pro Woche kürzer zu arbeiten. Mit durchschnittlich 44,5 Std. (West) bzw. 45,2 Std. (Ost) lagen deren tatsächliche wöchentliche Arbeitszeiten 2009 bereits auf dem Niveau, das die Bundesagentur für Arbeit mit ihren Modellrechnungen vorschlägt, um die befürchtete Verknappung des Arbeitskräfteangebots abzuwehren (siehe Abschnitt 2.2). Insofern geht der Vorschlag der BA, ungeachtet der inkompatiblen Arbeitszeitwünsche, zumindest bei vollzeitbeschäftigten Männern ins Leere und lässt keine zusätzlichen Potenzialeffekte erwarten.

Weniger deutlich als die tatsächlichen weichen die gewünschten von den vereinbarten Arbeitszeiten ab. Während ostdeutsche vollzeitbeschäftigte Männer durchschnittlich 0,3 Std. kürzer arbeiten möchten, favorisieren ihre westdeutschen Kollegen eine um 0,3 Std. längere Arbeitszeit als vereinbart. Geht man von Durchschnittswerten aus, dann treffen die vereinbarten Regelarbeitszeiten ziemlich genau die Zeitwünsche der vollzeitbeschäftigten Männer, nicht aber deren tatsächliche Arbeitszeiten. Gelänge es, die tatsächliche Dauer der Arbeitszeit mit der vertraglichen in Einklang zu bringen, würde ein solcher Schritt den Zeitnutzen vollzeitbeschäftigter Männer erheblich steigern können. Allerdings würden die durchschnittlich verkürzten Arbeitszeiten das Angebotspotenzial weiter verknappen, falls dieser Effekt nicht durch geringere Krankenstände und längeren Verbleib im Erwerbsleben aufgrund geringerer Arbeitsbelastungen kompensiert wird.

ABB.

\section{Vereinbarte, tatsächliche und gewünschte Arbeitszeit nach Geschlecht in Deutschland 1993 - 2009}

in Wochenstunden

Frauen $\quad$ tatsächlich $\square$ vereinbart $\square$ gewünscht

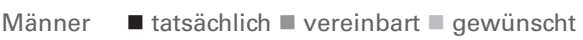

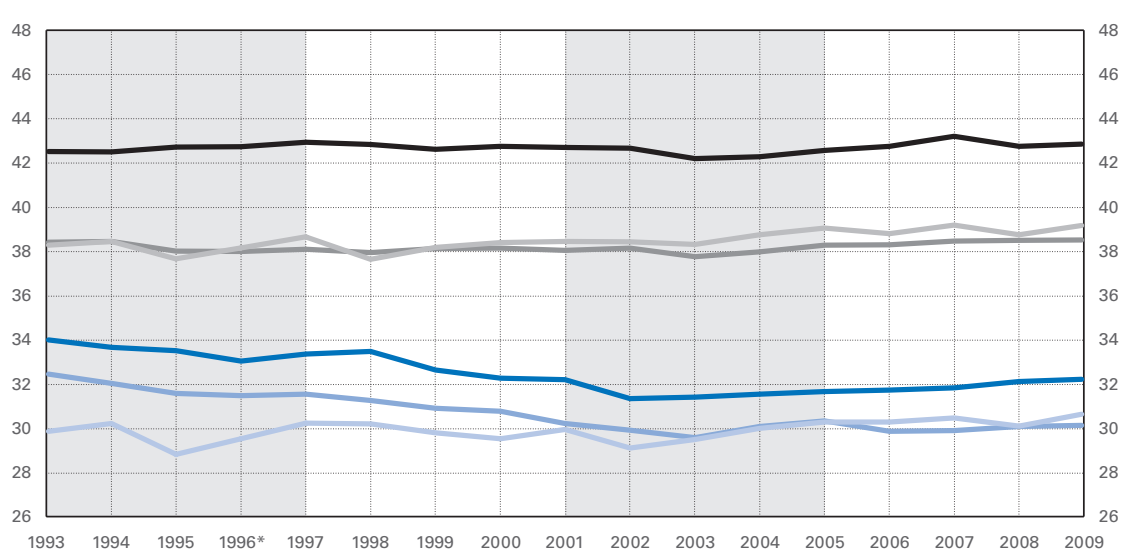

*Für 1996 liegen keine Angaben zu den gewünschten Arbeitszeiten vor.

Phasen der Eintrübung auf dem Arbeitsmarkt sind grau unterlegt.

Quelle: SOEP 1993-2009; Berechnung und Darstellung der Autoren.

\subsection{Teilzeitbeschäftigte}

Auch bei Teilzeitbeschäftigten - in der weit überwiegenden Zahl Frauen - stimmen die drei Größen der Arbeitszeitdauer nicht überein. ${ }^{8}$ Zudem zeigen sich erhebliche Niveauunterschiede zwischen Ost- und Westdeutschland. Alle drei Zeitgrößen übertreffen in den neuen Bundesländern deutlich die Vergleichswerte in den alten Ländern (Abbildung 2). Ostdeutsche teilzeitbeschäftigte Frauen arbeiten mit 27,2 Std. durchschnittlich über 6 Std. länger als ihre westdeutschen Kolleginnen. Offensichtlich wirkt die stärkere Orientierung von ostdeutschen Frauen an der in der DDR üblichen Vollzeitarbeit im Rahmen des Zweiverdienermodells weiter nach (Klenner 2009). Eine Rolle kann aber auch das immer noch bestehende Lohngefälle zwischen Ost und West spielen sowie der aufgrund der auch bei Männern höheren Arbeitslosenquote wachsende Anteil von Familienernährerinnen (ebd.).

In beiden Teilen Deutschlands gehen die durchschnittlich gewünschten Arbeitszeiten nicht nur deutlich über die vereinbarten, sondern auch über die tatsächlichen Werte hinaus. In den alten Bundesländern wollten 2009 die Frauen ihre vereinbarten um 4,1 und ihre tatsächlichen Arbeitszeiten um 3 Std. erhöhen; in Ostdeutschland lauten die Vergleichswerte 5,2 und 2,9 Std. Auffallend ist, dass die tatsächliche durchschnittliche Arbeitszeit in Westdeutschland seit Inkrafttreten des TzBfG auch bei den teilzeitbeschäftigten Frauen unverändert geblieben ist. Die Werte der gewünschten Zeiten haben jedoch zugelegt. Nicht ganz so deutlich vergrößert haben sich die Zeitdiskrepanzen auch in Ostdeutschland. Offensichtlich hat das TzBfG diese Entwicklungen nicht verhindert. Der rechtliche Anspruch, von Vollzeit zu Teilzeit wechseln zu können, scheint eine notwendige, aber längst nicht auch hinreichende Bedingung zu sein, dass Teilzeitbeschäftigte ihre gewünschten Arbeitszeiten auch tatsächlich realisieren.

\subsection{Zwischenfazit}

Die tatsächlichen Arbeitszeiten entsprechen, legt man Durchschnittswerte zugrunde, längst nicht den Geld-Zeitpräferenzen der Beschäftigten. Besonders groß sind die Diskrepanzen bei vollzeitbeschäftigten Männern in Westund in Ostdeutschland sowie bei teilzeitbeschäftigten Frauen in Ostdeutschland. Die Wünsche weisen jedoch in unterschiedliche Richtungen. Während, immer gemessen an Durchschnittswerten, vollzeitbeschäftigte Frauen und Männer in Ost- wie in Westdeutschland kürzer arbeiten möchten, besteht bei teilzeitbeschäftigten Frauen der Wunsch nach längeren Arbeitszeiten. 


\section{Vereinbarte, tatsächliche und gewünschte Arbeitszeit vollzeitbeschäftigter Frauen und Männer in West- und Ostdeutschland $1993-2003$}

Frauen

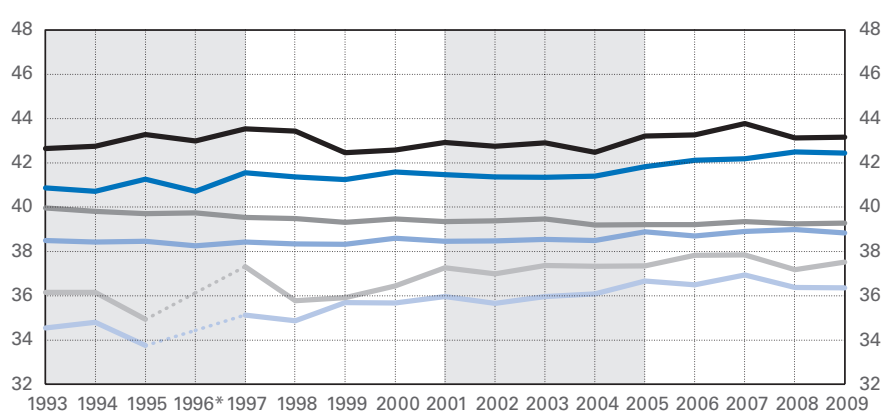

Männer

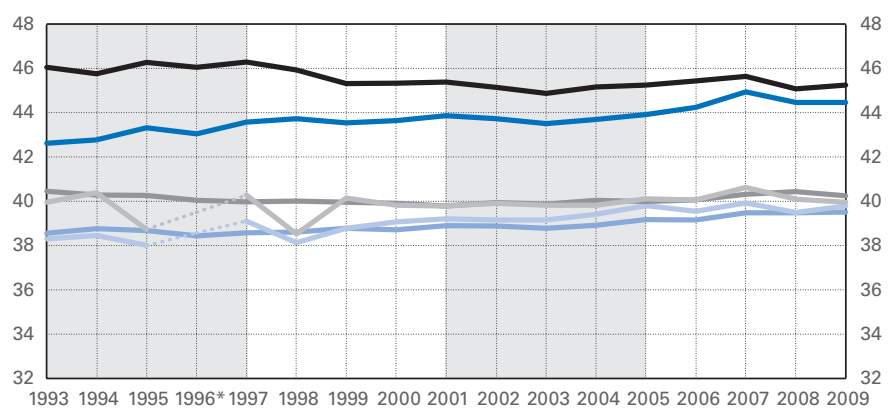

*Für 1996 liegen keine Angaben zu den gewünschten Arbeitszeiten vor. Phasen der Eintrübung auf dem Arbeitsmarkt sind grau unterlegt.

\section{Arbeitszeitwünsche und Beschäftigungseffekte}

Wenn tatsächliche und gewünschte Arbeitszeiten stark auseinanderklaffen, stellt sich die Frage, welche Wirkungen es für den Arbeitsmarkt hätte, wenn die Beschäftigten präferenzgerecht arbeiten könnten. Gegen den Realitätsgehalt derartiger Modellrechnungen lässt sich einwenden, dass sie nicht die tatsächlichen Entscheidungssituationen der Beschäftigten simulieren können, lediglich auf Aussagen im Rahmen von letztlich unverbindlichen, nicht entscheidungsrelevanten Befragungen beruhen, Rückkoppelungen auf Arbeitszeitwünsche und -entscheidungen von Partnerinnen und Partnern nicht berücksichtigen und auf die Nagelprobe verzichten müssen. Ebenso wenig bleibt im Kalkül berücksichtigt, inwieweit das Nachfrageprofil in räumlicher, qualifikatorischer und auch zeitlicher Hinsicht mit den Zeitwünschen kompatibel ist. Völlig ausgeblendet bleiben ökonomische Effekte auf Produktivität, Einkommen, privaten Konsum usw. und damit auf wirtschaftliches Wachstum und Beschäftigung. Deshalb können derartige Modellrechnungen lediglich grobe Anhaltspunkte liefern. In jeden Fall aber können sie anzeigen, in welche Richtung sich das Angebotspotenzial ändern wird und welche maximalen Volumina in etwa zu erwarten sind.

Bilanziert man rechnerisch die Zahl der Beschäftigten, die eine Änderung ihrer Arbeitszeiten wünschen, und deren jeweilige Änderungsvolumina, so überwiegt im Saldo deutlich das Verkürzungspotenzial (Holst 2010). Starke Unterschiede zeigen sich auch 2009 zwischen Männern und Frau- en, zwischen Ost und West und je nach aktuell geleisteter Arbeitszeitdauer (Abbildungen 3 und 4). Nicht überraschend ist der Befund, dass unter denjenigen mit kurzen Arbeitszeiten der Wunsch nach längerer Arbeit dominiert. Wer lange arbeitet, plädiert umgekehrt für vermehrte Zeit zur eigenen Verfügung. Je länger die Arbeitszeit, desto größer ist der Verkürzungswunsch, und umgekehrt steigt der Verlängerungswunsch mit abnehmender Dauer der bisherigen Arbeitszeit. Anders formuliert: Die Unzufriedenheit über die gegebene Arbeitszeit ist besonders ausgeprägt an den Rändern, wo entweder sehr kurz oder sehr lang gearbeitet wird.

Männer streben eher kürzere und Frauen eher längere Arbeitszeiten an. Bei den Frauen lassen sich bei den Wunscharbeitszeiten zwei Schwerpunkte identifizieren, die zwischen 30 und 35 Std. (Ost: knapp $38 \%$ und West: knapp $30 \%$ ) sowie um die 40 Std.-Marke (Ost: $34 \%$ und West: knapp $18 \%$ ) liegen. Für längere Arbeitszeiten plädieren vor allem Frauen in Westdeutschland mit tatsächlichen Arbeitszeiten von weniger als 30 Std. Deren Zahl übersteigt deutlich die mit den umgekehrten Wünschen (Abbildung 3). Diese Teilgruppe, auf die knapp $40 \%$ der westdeutschen Frauen entfallen, ist für fast $70 \%$ des gesamten rechnerischen Volumens verantwortlich, das durch Arbeitszeitverlängerungen zustande käme. In Ostdeutschland überwiegen die Wünsche nach längeren Arbeitszeiten bei Frauen, die bis zu 35 Std. arbeiten. Nur diejenigen mit längeren Arbeitszeiten votieren für kürzere. Vor allem aber Männer mit langen Arbeitszeiten von mehr als $40 \mathrm{Std}$. sprechen sich in Ost- und Westdeutschland für teilweise beträchtliche Ausweitungen der nicht erwerbsgebundenen Zeiten aus 
ABB. 3

Vereinbarte, tatsächliche und gewünschte Arbeitszeit teilzeitbeschäftigter Frauen in West- und Ostdeutschland 1993 - 2009 in Wochenstunden Westdeutschland $\quad$ tatsächlich $\square$ vereinbart $\square$ gewünscht Ostdeutschland

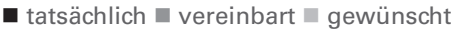

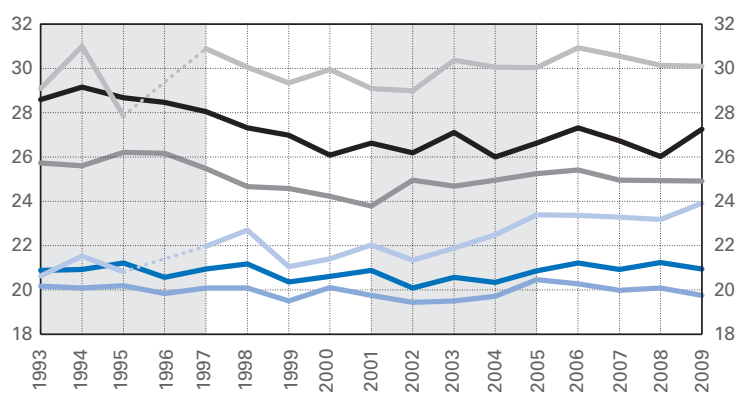

*Für 1996 liegen keine Angaben zu den gewünschten Arbeitszeiten vor. Phasen der Eintrübung auf dem Arbeitsmarkt sind grau unterlegt.

Quelle: SOEP 1993-2009; Berechnung und Darstellung der Autoren.

(Abbildung 4). Zu dieser Gruppe gehört über die Hälfte der Männer (Ost: 57 \%, West: $55 \%$ ).

Insgesamt errechnet sich aus den Arbeitszeitwünschen ein Verkürzungsvolumen, das in etwa 2,2 Mio. Vollzeitäquivalenten (auf Basis der durchschnittlichen tariflichen Wochenarbeitszeit von 37,7 Std.) entspricht. Selbst wenn man unterstellt, dass gewünschte Arbeitszeiten, die um weniger oder mehr als 2 Std. von den tatsächlichen abweichen, als durchaus präferenzgerecht anzusehen und nur die darüber hinausgehenden Zeitwünsche zu berücksichtigen sind, errechnet sich noch ein Potenzial von gut 2 Mio. Vollzeitäquivalenten. Rechnerisch übertreffen die Werte des gewünschten Verkürzungspotenzials, gemessen in Vollzeitäquivalenten, mit minus ca. 3,7 Mio. die der Verlängerungswünsche mit plus ca. 1,5 Mio. um mehr als das Doppelte.

Eine Arbeitszeitgestaltung, die sich an den Zeitpräferenzen aller Beschäftigten orientiert und versucht, deren Zeitnutzen zu steigern, erscheint zumindest rein rechnerisch nicht geeignet, das Angebotspotenzial zu steigern. Allerdings lassen derartige Modellrechnungen interdependente Reaktionen und Verhaltensänderungen außer Acht. So ist denkbar, dass in erwerbstätigen Paarhaushalten Änderungen in der Arbeitszeitdauer eines Partners nicht ohne Einfluss auf die Arbeitszeitdauer des anderen bleiben. Keineswegs auszuschließen ist dabei, dass unter Abwägung des zeitlichen und monetären Haushaltsbudgets und dessen Neuaufteilung zwischen den Partnern das gesamte Kürzungsvolumen der Arbeitszeit geringer ausfällt als in den hier skizzierten Modellrechnungen ausgewiesen. Bei den abgefragten Zeitwünschen handelt es sich um Individualperspektiven, die nicht die Haushaltskalküle und -präferenzen reflektieren.

Denkbar ist ferner, dass präferenzgerechtere Arbeitszeiten einen Sog ausüben auf bislang nicht erwerbstätige Frauen, vor allem auf Mütter mit Kindern bis 16 Jahre. Modellrechnungen schätzen das aktivierbare Potenzial auf fast 1,5 Mio. Vollzeitäquivalente (Eichhorst et al. 2011). Wenngleich von diesem unter Idealbedingungen hinsichtlich Betreuungsinfrastruktur, Arbeitszeit und Erwerbsbereitschaft geschätzten Wert realiter Abstriche zu machen sind, so zeigen die Berechnungen doch, dass ein nicht unbeachtliches Arbeitskräftepotenzial existiert, das sich mit familienfreundlicheren, kurzen Arbeitszeitmustern aktivieren ließe.

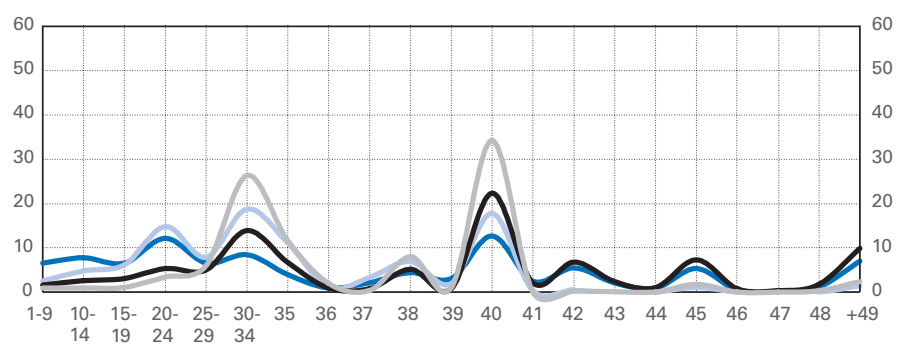

Männer

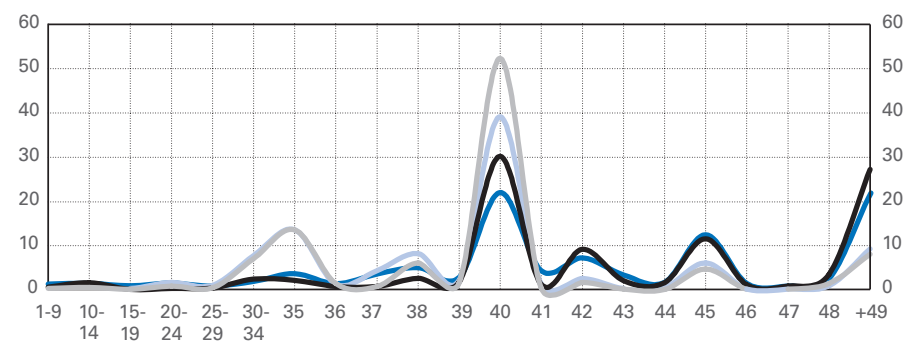


Eine präferenzgerechte Arbeitszeitgestaltung lässt außerdem einen längeren Verbleib im Erwerbsleben erwarten. Kürzere Arbeitszeiten gerade in Bereichen mit hoher Arbeitsbelastung versprechen nicht nur den Krankenstand zu senken, sondern auch die Zahl der Rentenzugänge wegen Erwerbsminderung zu verringern und das durchschnittliche Rentenzugangsalter zu erhöhen. Positive Potenzialeffekte wären die Folge. Insofern sind Befürchtungen, präferenzgerechte Arbeitszeiten könnten zukünftige Knappheit beim Arbeitsangebot verschärfen, zumindest zu relativieren, wenn nicht gar als weitgehend unbegründet einzustufen.

\section{Fazit}

Faktische, vereinbarte und gewünschte Arbeitszeiten klaffen auseinander. Vollzeitbeschäftigte Männer und Frauen wünschen eher kürzere, teilzeitbeschäftigte Frauen eher längere Arbeitszeiten. Die Arbeitszeitwirklichkeit ist noch weit von einem nutzenmaximalen Verhältnis von Einkommen und nicht-erwerbsgebundener Zeit entfernt. Arbeitszeitpolitischer Handlungsbedarf besteht nach wie vor. Für die Beschäftigten würde sich der Zeitnutzen erheblich steigern lassen, wenn es gelänge, die tatsächlichen mit den individuell oder kollektiv vereinbarten Regelarbeitszeiten stärker in Übereinstimmung zu bringen. Eine solche enger an den Zeitpräferenzen der Beschäftigten orientierte Zeitpolitik hat nicht nur differenzierten Zeitwünschen Rechnung zu tragen, sondern muss auch offen sein für im Zeitablauf sich ändernde Bewertungen des Geld-Zeitnutzens. Diese doppelten Anforderungen versprechen Wahlarbeitszeiten zu erfüllen, wie sie bereits das Konzept der „garantierten Optionalität“ (Hinrichs 1988) vorgeschlagen hat. Der Grundgedanke besteht darin, den Beschäftigten Rechte einzuräumen, je nach persönlichen Lebenslagen und individuellen Bedürfnissen von der Regelarbeitszeit abweichen zu können, ohne jedoch deren Schutz aufzugeben. Die in der tariflichen Regelarbeitszeit angelegte Schutzfunktion wäre um eine Gestaltungsfunktion zu erweitern, wie sie ansatzweise im Teilzeitund Befristungsgesetz angelegt ist.

Ein auf Wahlarbeitszeiten basierendes Konzept würde zweifellos die Realisierungschancen für zeitpolitische Zielvielfalt verbessern und erlauben, sowohl den Zielen des alternsgerechten Arbeitens wie dem nach verbesserter Work-Life-Balance, familiengerechter Zeitgestaltung und erst recht dem nach vermehrter Zeitsouveränität besser Rechnung zu tragen. In dem Maße, wie es gelingt, diese Ziele für Frauen und für Männer einzulösen, ist auch mit positiven Effekten für das Erwerbspersonenpotenzial zu rechnen. Arbeitskräfte können ihre Beschäftigungsfähigkeit länger sichern; passgenauere Zeitarrangements versprechen, bislang nicht oder nur in geringerem Umfang erwerbstätige Frauen für den Arbeitsmarkt stärker zu mobilisieren. Insofern stellen Wahlarbeitszeiten auch im Hinblick auf Effekte beim Arbeitskräfteangebot eine zielführende Alternative zu generellen Arbeitszeitverlängerungen dar.

\section{LITERATUR}

Bell, D./Otterbach, S./Sousa-Poza, A. (2011): Work Hours Constraints and Health, IZA Discussion Paper (6126), Bonn

Bundesagentur für Arbeit (BA) (2011): Perspektive 2025: Fachkräfte für Deutschland, Nürnberg

Bundesministerium für Familie, Senioren, Frauen und Jugend (BMFSFJ)

(2005): Fünfter Bericht zur Lage der älteren Generation in der Bundesrepublik Deutschland, Berlin

Bundesministerium für Familie, Senioren, Frauen und Jugend (BMFSFJ)

(2011): Zeit für Familie. Ausgewählte Themen des Achten Familienberichts. Monitor Familienforschung, Ausgabe 26, Berlin

Deutsche Rentenversicherung (DRV) Bund (2011): Rentenversicherung in Zeitreihen, Berlin

Eichhorst, W./Marx, P./Tobsch, V. (2011): Familienfreundliche flexible Arbeitszeiten - ein Baustein zur Bewältigung des Fachkräftemangels, IZA Research Report (33), Bonn

Groß, H./Seifert, H. (2010) (Hrsg.): Zeitkonflikte. Renaissance der Arbeitszeitpolitik, Berlin

Herzog-Stein, A./Seifert, H. (2010): Der Arbeitsmarkt in der Großen Rezession Bewährte Strategien in neuen Formen, in: WSI-Mitteilungen 63 (11), S. 551-559, http://www.boeckler.de/wsimit_2010_11_herzog-stein.pdf Hinrichs, K. (1988): Motive und Interessen im Arbeitszeitkonflikt, Frankfurt a. M Holst, E. (2010): Arbeitszeitwünsche und -realität von abhängig beschäftigten Frauen und Männern in West- und Ostdeutschland, in: Groß, H./Seifert, H. (Hrsg.): Zeitkonflikte. Renaissance der Arbeitszeitpolitik, Berlin

Klenner, C. (2009): Wer ernährt die Familie? Erwerbs- und Einkommenskonstellationen in Ostdeutschland, in: WSI-Mitteilungen 62 (11), S. 619-626, http:// www.boeckler.de/wsimit_2009_11_klenner.pdf

Kroh, M. (2010): Short-Documentation of the Update of the SOEP-Weights, 1984-2008, Deutsches Institut für Wirtschaftsforschung Berlin, Data Documentation Nr. 50, http://www.diw.de/documents/publikationen/73/diw_01.c.359697. de/diw_datadoc_2010-050.pdf

Seifert, H. (2005) (Hrsg.): Flexible Zeiten in der Arbeitswelt, Frankfurt a. M./ New York

Sachverständigenkommission zur Erstellung des Ersten Gleichstellungsberichtes der Bundesregierung (2011): Neue Wege - Gleiche Chancen. Gleichstellung von Frauen und Männern im Lebensverlauf, Gutachten an das Bundesministerium für Familie, Senioren, Frauen und Jugend, Berlin Statistisches Bundesamt (2011): Fachserie1, Reihe 4.1.2, Wiesbaden Wagner, G. G./Frick, J. R./Schupp, J. (2007): The German Socio-Economic Panel Study (SOEP) - Scope, Evolution and Enhancements, in: Schmollers Jahrbuch 127 (1), S. 139-169

Wanger, S. (2011): Viele Frauen würden gerne länger arbeiten, in: IABKurzbericht (9), Nürnberg

\section{AUTOREN}

ELKE HOLST, PD Dr., Forschungsdirektorin Gender Studies und Senior Economist am Deutschen Institut für Wirtschaftsforschung (DIW) Berlin / Längsschnittstudie Sozio-oekonomisches Panel. Arbeitsschwerpunkte: Labor and Gender Economics, insbesondere Gender Gaps in Führungspositionen, Verdiensten und der Arbeitszeit.

eholst@diw.de

HARTMUT SEIFERT, Dr., ehemaliger Leiter des Wirtschafts- und Sozialwissenschaftlichen Instituts (WSI) in der Hans-Böckler-Stiftung. Arbeitsschwerpunkte: Flexibilität am Arbeitsmarkt, Arbeitszeitforschung, Strukturwandel der Beschäftigung.

h.g.seifert@t-online.de 